\title{
Detection of Aromatic/Aliphatic Hydrocarbons and Amines by Laser-lon Mobility Spectrometry (LIMS)
}

\author{
C. Oberhüttinger, A. Langmeier, H. Oberpriller, M. Kessler, J. Göbel, G. Müller \\ EADS Innovation Works Germany \\ D-81663 München
}

\begin{abstract}
Trace detection of hydrocarbons has been investigated using a laser ion mobility spectrometer (LIMS). The LIMS instrument used employs resonant two-photon ionization as a mechanism for the generation of analyte gas ions. We find that LIMS easily ionizes aromatic and aliphatic hydrocarbons with conjugated and delocalized $\mathrm{C}-\mathrm{C}$ double bond systems. Another class of organic compounds that could be successfully detected was amines. These latter substances contain double-occupied lone pair electrons localized on basic $\mathrm{N}$ atoms. All substances successfully detected feature a sizeable UV absorption within a certain range of photon energies $E_{p h}$. Two-photon ionization is observed in case the molecule-specific ionization energy $E_{1}$ complies with the energetic condition $2 E_{p h}>E_{1}$.
\end{abstract}

\section{Introduction}

Ion Mobility Spectrometry (IMS) is increasingly used in the field of process analytics and gas analysis [1]. In IMS, analyte gas molecules are ionized and accelerated by an electric field towards a collector electrode. Different kinds of molecules are distinguished by their specific drift times in the background air. State of the art IMS commonly employ radioactive ionization sources for the generation of analyte gas ions [2]. A shortcoming of radioactive IMS is that, firstly, their operation is subject to special authority regulations and that, secondly, these instruments only exhibit moderate selectivity. The latter property reveals from the fact that radioactive radiation ionizes the huge concentrations of main air constituents alongside with the tiny concentrations of trace analyte gases to be detected. A more selective ionization can be achieved by employing two-photon laser ionization [3]. A molecule with ionization energy $E_{1}$ can be ionized if the energy $E_{p h}$ of two identical photons exceeds the ionization energy $\left(2 E_{p h}>E_{1}\right)$ and the lifetime of the intermediate state is large enough. The underlying process is illustrated in Fig. 1, showing that the first photon elevates the molecule to an intermediate state which has a certain lifetime $\tau$. Provided $\tau$ is large enough, the molecule can be ionized by absorbing a second laser photon. This process is a special case of a so-called resonance-enhanced multi-photon ionization (REMPI) process [4]. Gases which lend themselves very well to two-photon ionization are aromatic hydrocarbons [5]. In contrast, air constituents and air contaminants like e.g. $\mathrm{N}_{2}, \mathrm{O}_{2}, \mathrm{H}_{2} \mathrm{O}$, and $\mathrm{CO}_{2}$ cannot be ionized as their ionization energy is higher than the available excitation energy [6]. Many other molecules, which fulfil the condition $2 \mathrm{E}_{\mathrm{ph}}>\mathrm{E}_{\mathrm{l}}$, are not ionized because the lifetime of the intermediate states is not long enough to absorb a second photon while being excited.

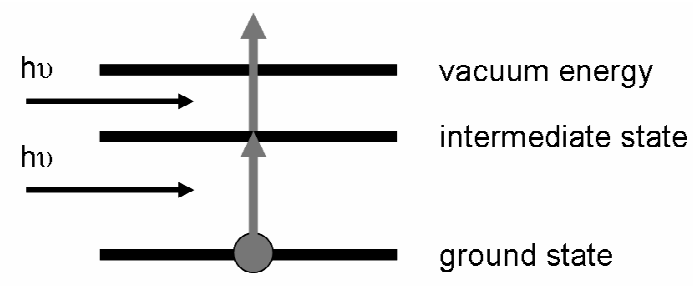

Fig.1: Molecular and continuum electronic levels involved in resonant two-photon ionization. 


\section{Hardware}

The measurements were performed with a planar drift tube, schematically shown in Fig. 2. This design was chosen as it provides a relatively long light-molecule interaction path. The gas molecules are guided into the drift tube where they get ionized by a laser beam. The so generated ions are accelerated as indicated towards a collector electrode where the ion current is measured and amplified. The utilized laser is a tuneable UV laser based on a flash-lamp pumped Nd:YAG crystal. The frequency is tripled to arrive at a wavelength of $355 \mathrm{~nm}$. After an optical parametric oscillator and an additional frequency doubling, wavelengths from 210 to $355 \mathrm{~nm}$ can be generated. A pulse energy of $50 \mu \mathrm{J}$ was used in our measurements. The laser had a pulse length of $5 \mathrm{~ns}$ and a spectral linewidth of $4-7 \mathrm{~cm}^{-1}$.

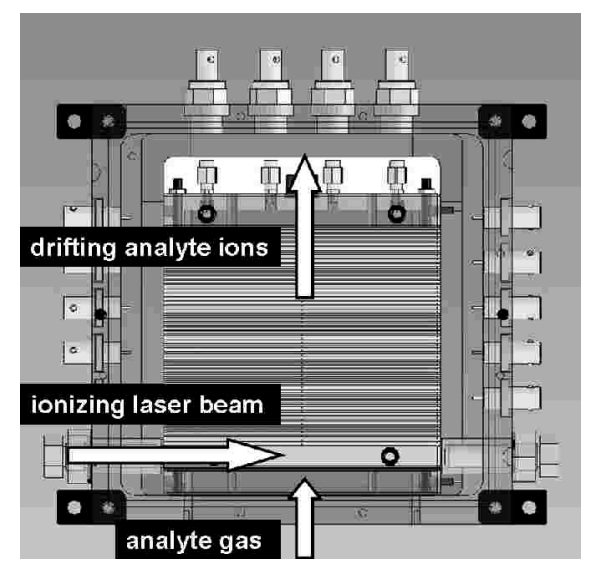

Fig.2: Schematic design of the utilized drift tube for ion mobility measurements.

The gas concentrations used varied in the range from 0.5 to $1 \mathrm{ppm}$ in the case of aromatic and aliphatic hydrocarbons. Amine concentrations were $2 \mathrm{ppm}$ in the case of methylamine and $3 \mathrm{ppm}$ in the cases of di-and trimethylamine. All substances were injected into the LIMS via a syringe device where they were vaporized. After vaporization, the substances were diluted to the required concentrations by adding a stream of nitrogen. The measurements were performed in the wavelength ranges extending from $210-$ $280 \mathrm{~nm}$ and $220-280 \mathrm{~nm}$, respectively, using a spectral step size of $2 \mathrm{~nm}$.

\section{Results}

With this experimental setup, different classes of molecules were investigated. A first interesting group are aromatic hydrocarbons. This class of molecules firstly exhibits a strong UV absorption in the photon energy range investigated and, secondly, their ionization energy is low enough to fulfil the condition $2 \mathrm{E}_{\mathrm{ph}}>\mathrm{E}_{\mathrm{l}}$. Altogether, nine aromatic hydrocarbons were studied with different ionization potentials ranging from $7.2 \mathrm{eV}$ to $9.9 \mathrm{eV}$. The IMS drift spectra of four of these compounds ( $p$-xylene, aniline, benzene, and nitrobenzene) are depicted in Fig. 3.

The following observations are relevant: (i) the intensity of the ion peak varies as the excitation wavelength is scanned, and (ii) the ion drift times are molecule-specific. Both criteria can be used to distinguish between the different kinds of gases. Whereas drift time is a classical criterion in IMS, the wavelength dependence of the ionization process appears as a second selectivity feature in LIMS. This latter fact clearly reveals from the molecule-specific $2 d$ patterns in Figs. 3a-d.

Considering the individual ionization spectra, we note that these, in principle, follow the corresponding single-photon UV absorption spectra. Fig. 4 illustrates this connection for the case of p-xylene. In this first case the match between the single-photon UV absorption and two-photon ionization current is very good, whereas in most other cases such a good match cannot be observed. One explanation is the existence of a long-wavelength cut-off which appears if the excitation energy is not sufficient to exceed the ionization energy. For an ionization energy of $E_{1}=10 \mathrm{eV}$, for instance, $\lambda_{c} \sim 250 \mathrm{~nm}$. Referring back to Fig. 3d, the poor ionization of nitrobenzene for $\lambda>230 \mathrm{~nm}$ can be explained in this way. Considering absolute magnitudes of the ionization current, we find that substances are more sensitively detected in case $2 E_{p h}$ significantly exceeds $E_{1}$. This, for instance, is the case for $p$-xylene and aniline. 

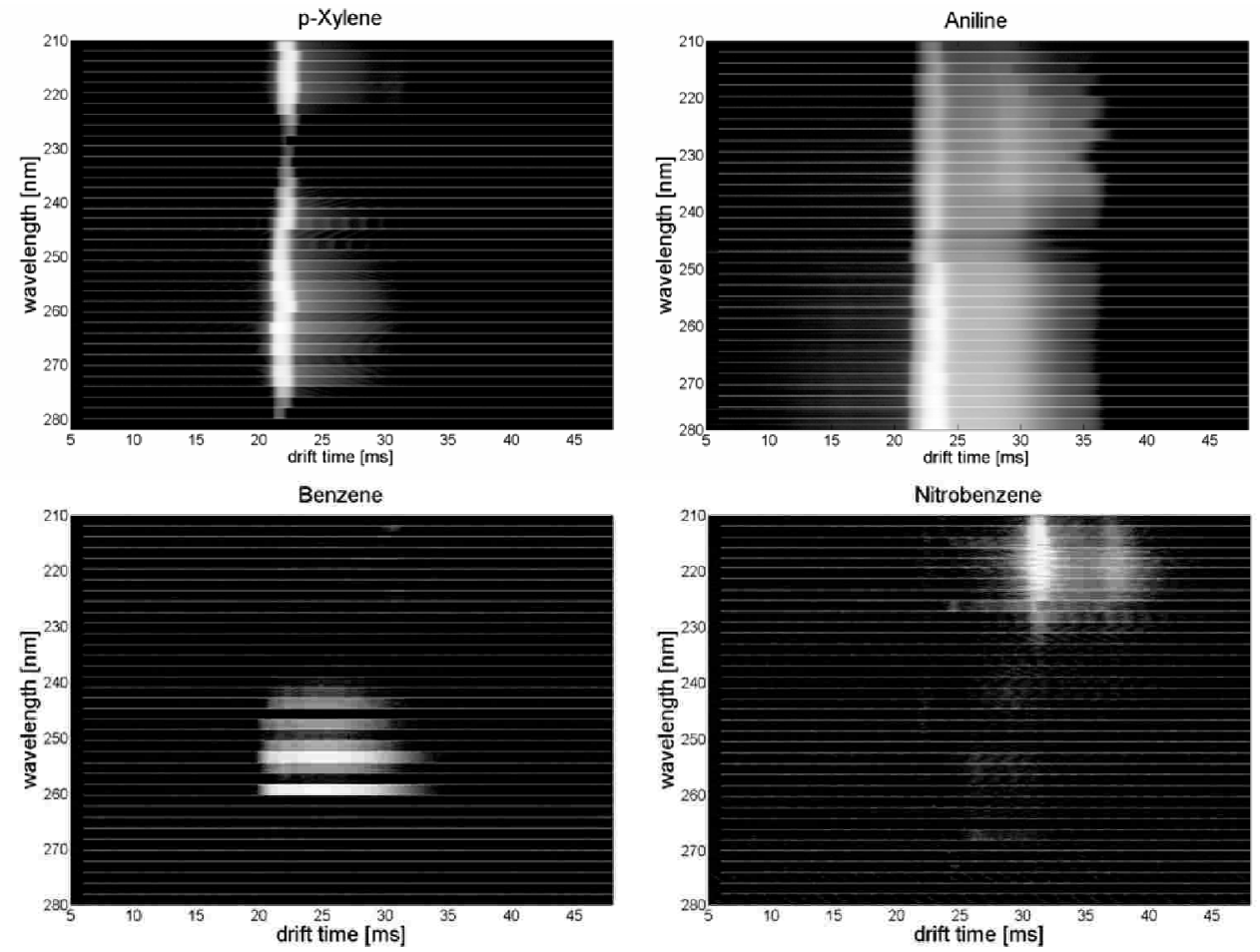

Fig.3: Drift time spectra of four aromatic hydrocarbons ((a) p-xylene, (b) aniline, (c) benzene, and (d) nitrobenzene) in the wavelength range extending from $210-280 \mathrm{~nm}$.

The aromatic hydrocarbons considered above share as a common property an electron system that is delocalized over the ring of conjugated $\mathrm{C}-\mathrm{C}$ double bonds in their molecular core structures. In order to assess the importance of such delocalized electron systems, our investigations were further extended to aliphatic hydrocarbons which do or do not exhibit electron delocalization. Within this latter class of hydrocarbons, three substances were chosen which all contain six carbon atoms but different numbers of single and double bonds in their $\mathrm{C}-\mathrm{C}$ backbone structures. These were: 2,4-hexadiene, 1-hexene, and nhexane. The ionization energies of these substances range from $8.48 \mathrm{eV}$ to $10.13 \mathrm{eV}$ and thus lie within the range of $E_{1}$ that should be accessible with the help of our laser source. In the case of $n$-hexane all $C$ atoms are connected by single $\sigma$-bonds; 1 -hexene, in contrast, contains an isolated double bond in a row of single bonds and 2,4-hexadiene a system of conjugated double bonds, i.e. single bonds alternating with double bonds. As an important result we observe that only this latter kind of molecule can be ionized. Again, this molecule stands out within its group in that it shows a significant single-photon absorption within the accessible photon energy range. As a proof for successful two-photon ionization we show in Fig. 5 the drift spectrum of 2,4-hexadiene as observed using a laser excitation wavelength of $220 \mathrm{~nm}$. In summary, electron delocalization again revealed as a decisive parameter with regard to successful twophoton ionization.

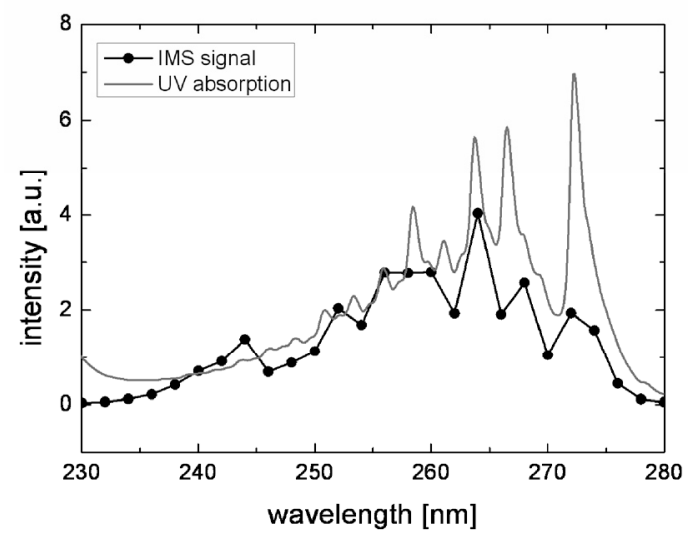

Fig.4: Comparison of IMS current signal and UV absorption spectrum of p-xylene in the wavelength range extending from $230-280 \mathrm{~nm}$. 


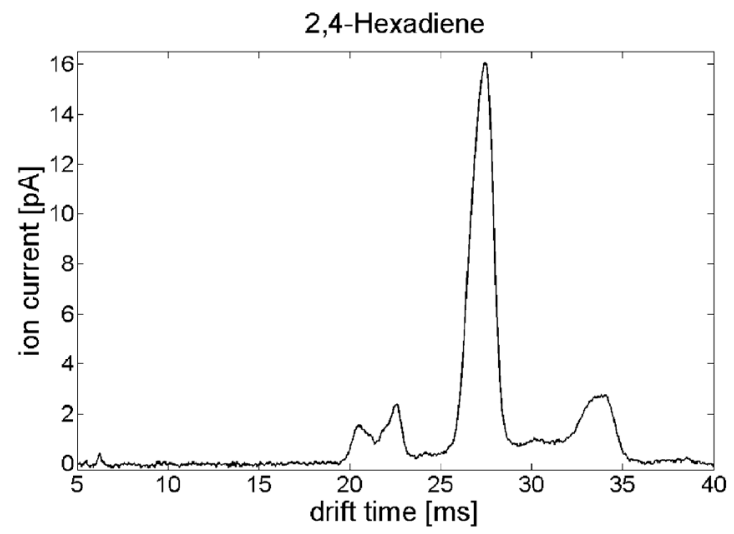

Fig.5: Ion drift spectrum of 1ppm of 2,4-hexadiene as observed at a laser excitation wavelength of $220 \mathrm{~nm}$.

Another interesting class of organic compounds are amines. In contrast to the hydrocarbons with delocalized and conjugated $\mathrm{C}-\mathrm{C}$ double bond systems, amines possess double-occupied lonepair electrons, which are attached to a basic nitrogen atom. As the amines investigated do exhibit particularly low ionization energies in the range of $8.9 \mathrm{eV}$ to $7.9 \mathrm{eV}$ and also a significant UV absorption in the accessible laser photon energy range, these latter substances suggested being good candidates for successful two-photon ionization. Measurements were therefore performed with $2 \mathrm{ppm}$ of methylamine and $3 \mathrm{ppm}$ of di- and trimethylamine in the wavelength range extending from $220 \mathrm{~nm}$ to $280 \mathrm{~nm}$. Two exemplary drift spectra, obtained at a laser excitation wavelength of $220 \mathrm{~nm}$, are shown in Fig. 6. These spectra exhibit clear peaks at a drift times of $21 \mathrm{~ms}$ and $23 \mathrm{~ms}$, respectively, with the larger dimethylamine molecule exhibiting the longer ion drift time.
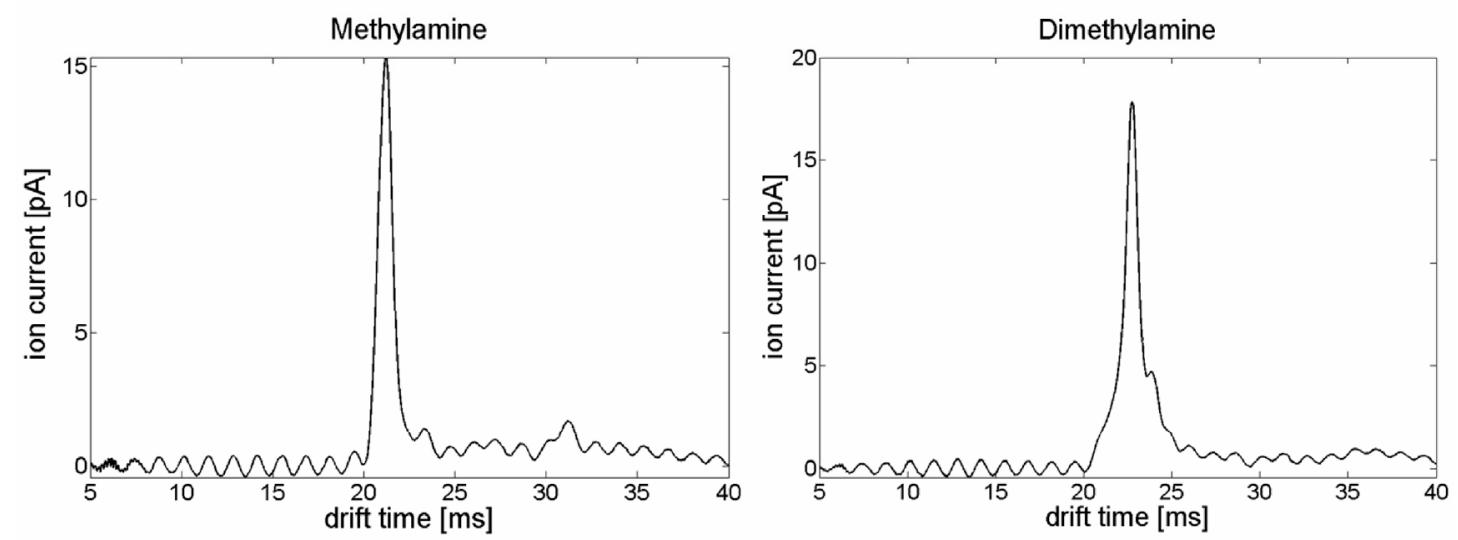

Fig.6: Drift spectra for (left) 2 ppm of methylamine and (right) 3 ppm of dimethylamine as observed using a laser excitation wavelength of $220 \mathrm{~nm}$.

\section{Conclusions}

Hydrocarbon detection was investigated using a laser ion mobility spectrometer (LIMS) with resonant twophoton ionization as the principle ionizing mechanism. Comparative measurements of the single-photon UV absorption and of the two-photon ionization probability were carried out to assess conditions under which substances can be successfully detected using resonance-enhanced two photon laser ionization. We find that LIMS does neither ionize the main constituents of the ambient air $\left(\mathrm{N}_{2}, \mathrm{O}_{2}, \mathrm{H}_{2} \mathrm{O}, \mathrm{CO}_{2}\right)$ nor most air contaminants $\left(\mathrm{CO}, \mathrm{NO}, \mathrm{NO}_{2}, \mathrm{O}_{3}\right)$ or single-bonded hydrocarbons. Primary ionization is largely restricted to aromatic and aliphatic hydrocarbons with delocalized $\mathrm{C}-\mathrm{C}$ double bond systems. Direct ionization could also be observed with hydrocarbons containing amino groups. These low-ionizationenergy molecules contain double occupied lone-pair orbitals localized on basic $\mathrm{N}$ atoms. For all substances successfully detected, a strong dependence between the two-photon ionization current and the single-photon UV absorption could be observed. 


\section{References}

[1] Baumbach J (2006) "Process analysis using ion mobility spectrometry", Anal Bioanal Chem, Vol. 384, pp.1059

[2] Eiceman GA, Karpas Z (2005) Ion Mobility Spectrometry. CRC Press, Boca Raton

[3] Lubman DM, Kronick MN (1983) "Resonance-Enhanced Two-Photon Ionization Spectroscopy in Plasma Chromatography", Anal Chem, Vol. 55, pp.1486

[4] Löhmannsröben HG, Beitz T et al (2004) "Laser-based ion mobility spectrometry for sensing of aromatic compounds", Proc SPIE, Vol. 5547, pp. 16

[5] Boesl U, Zimmermann R et al (1994) "Resonance-Enhanced Multi-Photon lonization: A Species Selective Ion Source for Analytical Time-of-Flight Mass Spectroscopy", Chemosphere, Vol. 29, pp. 1429

[6] Oberhuettinger C, Langmeier A et al (2008) "Hydrocarbon Detection Using Laser Ion Mobility Spectrometry", Int J Ion Mobil Spectrom, (submitted) 